\title{
Chinese Handwriting Identification Based on Stable Spectral Feature of Texture Images
}

\author{
Yuchen Yan* Qinghu Chen Wei Deng Feng Yuan \\ School of Electronic Information, Wuhan University, Hubei 430079, China \\ *Corresponding author's E-mail address: yuchenyan@163.com
}

\begin{abstract}
In this paper, a spectral feature extraction method based on fast Fourier transformation for writer identification is presented. According to the constructibility of the texture image of handwriting, we put forward an estimation method for mathematical expectation value of texture image's spectral features. This method eliminates the randomness of spectral features and gets stable spectral features. The experiment results show that this approach enhances the identification accuracy to a large extent using data sets with large handwriting samples.
\end{abstract}

Keywords: Writer identification; Texture; Spectrum; Feature fusion

\section{Introduction}

Texture analysis is a widely used method in the field of image processing, analysis and identification. General methods implemented to obtain textural features mainly include multi-channel Gabor filter technique, the gray scale co-occurrence matrix (GSCM), wavelet transformation and so on [1-4]. As the most important method in text-independent writer identification, texture analysis has been widely applied to handwriting identification. In reference [5] multi-channel Gabor filter is employed to extract textural features and the highest identification accuracy is up to $95.7 \%$ using handwritings from 17 different people. In reference [6] Independent Component Analysis is adopted and the highest identification accuracy of $91.3 \%$ is achieved in a database of 30 people. And in reference [7] run-length measurement is used. By using a database of 19 people the highest accuracy is 91.3\%. In reference [8] Gabor filter is used for feature extraction, after which multi-class SVM is used to train and test the data from 87 persons, and it achieves the highest accuracy of $97.7 \%$ concerning Top-8.

However, all these textural methods mentioned above are only evaluated using data sets with rather small handwriting samples. In the real application, a writer identification system often performs one-to-many searches in a large database with at least hundreds of handwriting samples or even more. We notice that the normally used texture methods can perform well when the samples in the database are rather small. While the samples of handwritings in the database increase, the identification accuracy declines sharply. This raises a question: how to improve the accuracy of writer identification in large data set of handwriting.

To improve the identification accuracy in a writer identification system with large database, this paper presents a new method based on stable spectral features of texture. The method involves: 1) for a certain hand-written sample, which is firstly scanned to generate an image, we segment the whole image to get single Chinese characters; 2) Each single Chinese character will be normalized and then we randomly choose some of them to construct the texture images; 3) Fast Fourier Transformation (FFT) is used to extract spectral features and then the spectral features are fused so as to obtain stable features; 4) Finally, classification is implemented. We carry out experiments using data sets with handwriting samples collected from 100 persons and 
500 persons respectively and the results obtained are exciting and reasonable. Our method achieves the highest accuracy of $98 \%$ and $95 \%$ concerning Top-10 respectively. The experimental results show that this approach is very promising for text-independent handwriting identification.

The proposed method can be described as the following flow chart (see Fig.1). The original image is processed to generate a binary image with black foreground and white background in a preprocessing phase. The single Chinese characters, which are called "character pictures" (character picture is the picture of a single Chinese character extracted from the binary image which is used for writer identification.) are obtained by the way of human-computer interaction. Then the computer chooses parts of the character pictures to form several texture images, after which FFT is employed to extract features. Then all the feature vectors are acquired and fused to get the stable estimation. Finally we adopt the Euclidean distance classifier for identification.

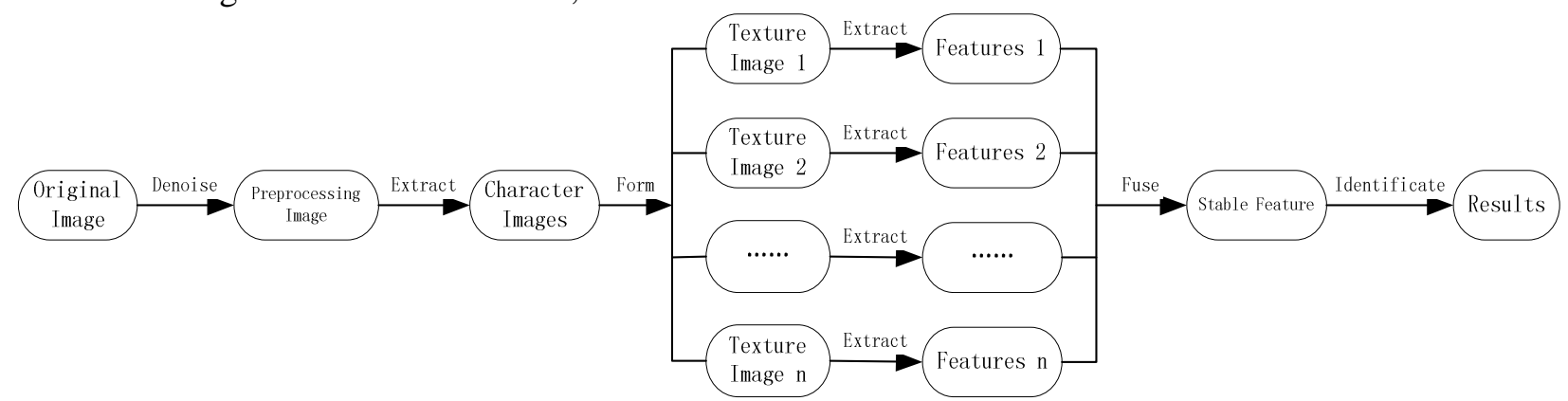

Figure.1 Flow Chart of proposed method

\section{Texture and it's spectral features}

\subsection{Texture constructing}

The procedure of constructing texture images:

Step 1: The acquisition of character pictures. The original image is scanned and the foreground/background separation can be realized in a preprocessing phase, yielding a white background with black ink. After the removal of noise, the single Chinese character is obtained with human aid which is called "character picture".

Step 2: The normalization of characters. To facilitate the identification all the characters should be normalized to a uniform size of $64 \times 64$ pixels and similar stroke width.

Step 3: the construction of texture image. Each texture image is formed in the way that the computer randomly chooses a hundred character pictures which are different from each other as possible as it can. Then each texture image is arranged with 10 rows and 10 columns. One sample of texture image is shown in Fig. 2.
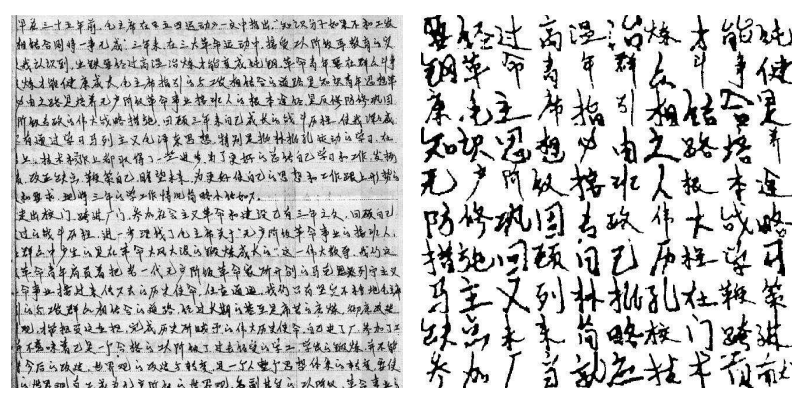

(a) Original image

(b) Texture image Figure. 2 An Example of texture images

\subsection{Spectral features extraction}

After the acquisition of texture images, FFT is applied for feature extraction. Actually FFT is a kind of multi-channel analysis method, namely, separating an image into several sub-images with different frequencies and directions and extracting features from these sub-images respectively. The feature extraction method in this paper can be specifically described as follows:

Suppose $f(x, y)$ represents a picture with size of $\mathrm{N} \times \mathrm{N}$ pixels, and the $2 \mathrm{D}$ FFT of this picture can be defined as:

$$
F(u, v)=\frac{1}{N} \sum_{x=0}^{N-1} \exp \left[\frac{-j 2 \pi u x}{N}\right] \sum_{y=0}^{N-1} f(x, y) \exp \left[\frac{-j 2 \pi v y}{N}\right]
$$

Where $u, v=0,1, \cdots, N-1, \quad x$ and $y$ are the spatial variables, and $u$ and $v$ are the frequency 
variables.

Since the 2D Fourier Transformation has separation property, it can be achieved by operating 1D Fourier Transformation twice.

$$
\begin{aligned}
& F(x, v)=N\left[\frac{1}{N} \sum_{y=0}^{N-1} f(x, y) \exp [-j 2 \pi v y / N]\right] \\
& F(u, v)=N\left[\frac{1}{N} \sum_{x=0}^{N-1} F(x, v) \exp [-j 2 \pi u x / N]\right]
\end{aligned}
$$

Where: $u, v=0,1, \cdots, N-1$.

We firstly discuss the 1D FFT. Actually the 1D Fourier Transform can be described as:

$$
F(u)=\frac{1}{N} \sum_{x=0}^{N-1} f(x) W_{N}^{u x}
$$

Where: $W_{N}=\exp [-j 2 \pi / N]$.

Suppose $N=2^{n}$, where $\mathrm{n}$ is a nonnegative integer.

$\mathrm{M}$ is a nonnegative integer, and $N=2 M$,

Then:

$$
\begin{aligned}
& F(u)=\frac{1}{2}\left[\frac{1}{M} \sum_{x=0}^{M-1} f(2 x) W_{M}^{u x}+\frac{1}{M} \sum_{x=0}^{M-1} f(2 x+1) W_{M}^{u x} W_{2 M}^{u}\right] \\
& F(u+M)=\frac{1}{2}\left[\frac{1}{M} \sum_{x=0}^{M-1} f(2 x) W_{M}^{u x}-\frac{1}{M} \sum_{x=0}^{M-1} f(2 x+1) W_{M}^{u x} W_{2 M}^{u}\right]
\end{aligned}
$$

The above equations show that a transformation with $\mathrm{N}$ dots can be divided into two parts, by which the computation cost is largely reduced. We zoom out the texture image to $512 \times 512$ pixels, so that the width and height of the image are both $2^{9}$. Finally the Fourier Transformation can be simplified to Fourier Transformation with two dots.

After FFT we get the frequency domain features $F(u, v)$. Actually the distribution of $F(u, v)$ can be shown in Fig. 3. After recombining the distribution in accordance with frequencies from low to high, a new distribution is shown in Fig. 4. which looks like a circle with low frequencies at the center of the circle and high frequencies at the edge of the circle.

Then for the recombined frequency domain features, we evenly divide the circle both in radial direction and angle direction to get a group of feature vectors representing the entropy distribution and direction characteristics. To illustrate it explicitly, we evenly divide the circle into nine parts in radial direction and eliminate the part nearest to the center of the circle. For the rest eight

parts, two of them which are neighboring each other are integrated into one part, that is, we only get four parts in radial direction; we evenly divide the circle into 120 parts in angle direction, as is shown in Fig. 5. Actually the whole circle is divided into $4 \times 120=480$ parts. As is well known, the distribution of FFT is symmetric, so only half of the information of the circle is needed to deal with. We calculate the expectation value of each small part, then a 240 dimensional vector is derived finally:

$$
\vec{X}=\left[X_{1}, X_{2}, \cdots, X_{M}\right]^{T}
$$

Where $M=240$.

\begin{tabular}{|l|l|}
\hline 4 & 3 \\
\hline 2 & 1 \\
\hline
\end{tabular}

Figure 3. Original Spectrum $F(u, v)$

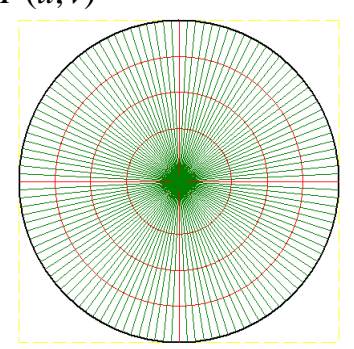

Figure 5. Divided Spectrum

\subsection{Distance and experiment}

After construction of texture images and the feature extraction, we assume that $\mathrm{X}$ represents the features of the handwriting for test and Y represents the features of the handwriting in the sample database.

$$
\begin{aligned}
& \vec{X}=\left[X_{1}, X_{2}, \cdots, X_{M}\right]^{T} \\
& \vec{Y}=\left[Y_{1}, Y_{2}, \cdots, Y_{M}\right]^{T}
\end{aligned}
$$

And, $\quad d=\sqrt{\sum_{i=1}^{M}\left(X_{i}-Y_{i}\right)^{2}}$

where $\mathrm{d}$ is defined as the distance between two handwritings.

Here $d$ means the similarity between two handwritings. And smaller $d$ implies greater possibility that the two handwritings are from the same person.

To test the feasibility of the method proposed 
above, we collect 200 handwritings from 100 different people. Each person has two handwritings, one is used to establish the sample database and the other is used for test. For these handwritings, no matter what the content of them and writing tools used, the only requirement is that all the handwritings should contain at least 200 characters. We construct a texture image for each sample handwriting and testing handwriting, extract the features, classify and identify. The results are shown in Table1.

Table 1. Experiment results

\begin{tabular}{|c|c|c|c|c|}
\hline Results & Top 1 & Top 3 & Top 5 & Top 10 \\
\hline $\begin{array}{c}\text { One texture, } \\
100 \text { persons }\end{array}$ & $55 \%$ & $71 \%$ & $79 \%$ & $87 \%$ \\
\hline
\end{tabular}

The results show that this method can achieve certain useful result but the accuracy is not high enough. It is mainly because of the fluctuation of the texture features which finally leads to the random difference between the handwriting for test and the sample handwriting. Principally there are three random factors:

1) Randomness of writing: Due to the different writing tools, writing environment and the different state of writers at different time, handwritings may vary randomly.

2) Randomness of content: In the real application the content of the handwritings can vary at a large scale. Since the limited size of texture images, a texture image cannot contain all the characters. Obviously different characters lead to the difference of texture features.

3) Randomness of position of the characters: According to the method of constructing texture images proposed above, the characters in texture image may be located randomly, which also influences the stability of the texture features.

\section{Multi-texture constructing and stable features estimation}

\subsection{Mathematical expectation value of features}

Due to the random factors mentioned above, we describe the feature vectors as follows:

$$
\begin{aligned}
& \vec{\mu}=E(\vec{X})=\left[E\left(X_{1}\right), E\left(X_{2}\right), \cdots, E\left(X_{M}\right)\right]^{T}=\left[\mu_{1}, \mu_{2}, \cdots, \mu_{M}\right]^{T} \\
& \vec{\lambda}=E(\vec{Y})=\left[E\left(Y_{1}\right), E\left(Y_{2}\right), \cdots, E\left(Y_{M}\right)\right]^{T}=\left[\lambda_{1}, \lambda_{2}, \cdots, \lambda_{M}\right]^{T}
\end{aligned}
$$

For a certain handwriting, $\vec{\mu}$ and $\vec{\lambda}$ are confirmed unknown parameters which should keep stable. If one can obtain the exact value of $\vec{\mu}$ and $\vec{\lambda}$, the writer identification should achieve ideal results. Nevertheless due to the limited amount of characters in texture images, it is impossible to determine the distribution function of the feature vectors, namely it is impossible to get the exact values of $\vec{\mu}$ and $\vec{\lambda}$.

In order to get stable features we assume that if several texture images can be constructed, we can get the features of each image, the stable features may be achieved through fusion which is realized by statistical method, namely the stable estimated values of $\vec{\mu}$ and $\vec{\lambda}$ may be obtained.

\subsection{Multi-texture Constructing}

During the procedure of constructing texture images, we repeat step3 in 2.1 to get a number of texture images. The texture images obtained are shown in Fig. 6.
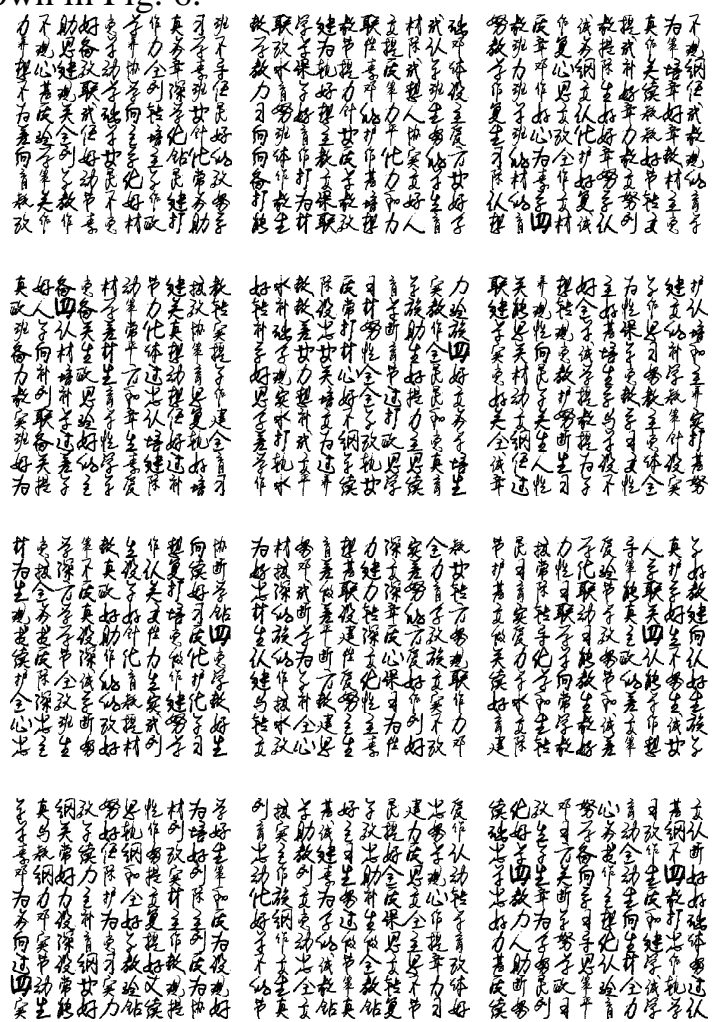

Figure 6. Multi-texture Constructing

\subsection{Stable features estimation}

Suppose that for each handwriting used for test there are s texture images, and the feature vectors are shown below:

$$
\vec{X}^{i}=\left[X_{1}^{i}, X_{2}^{i}, \cdots, X_{M}^{i}\right]^{T} \quad(i=1,2, \cdots, s)
$$

For each handwriting in the database there are $t$ texture images, and the feature vectors are shown 
below:

$$
\vec{Y}^{j}=\left[Y_{1}{ }^{j}, Y_{2}{ }^{j}, \cdots, Y_{M}^{j}\right]^{T} \quad(j=1,2, \cdots, t)
$$

Then we equilibrate the features as follows:

$$
\begin{aligned}
& \overrightarrow{\bar{X}}=\frac{1}{s_{i=1}^{s}} \sum^{i} \vec{X}^{i}=\left[\frac{1}{s} \sum_{i=1}^{s} X_{1}^{i}, \frac{1}{s_{i=1}} \sum_{i=1}^{s} X_{2}^{i}, \cdots ;-\frac{1}{s} \sum_{i=1}^{s} X_{M}^{i}\right]^{T}=\left[\bar{X}_{1}, \bar{X}_{2}, \cdots ; \bar{X}_{M}\right]^{T} \\
& \overrightarrow{\bar{Y}}=\frac{1}{t} \sum_{i=1}^{s} \vec{Y}^{j}=\left[\frac{1}{t} \sum_{j=1}^{t} Y_{1}^{j}, \frac{1}{t} \sum_{j=1}^{t} Y_{2}^{j}, \cdots,-\frac{1}{t} \sum_{j=1}^{t} Y_{M}^{j}\right]^{T}=\left[\bar{Y}_{1}, \bar{Y}_{2}, \cdots, \bar{Y}_{M}\right]^{T}
\end{aligned}
$$

$\overline{\vec{X}}$ and $\overline{\vec{Y}}$, acting as statistical mean parameters, are stable relatively. So they can be used to estimate $\vec{\mu}$ and $\vec{\lambda}$.

\subsection{Classifier design and it's performance}

\subsubsection{Euclidean distance classifier}

The Euclidean distance between $\vec{\mu}$ and $\vec{\lambda}$ is defined as:

$$
d_{e}=D(\vec{\mu}, \vec{\lambda})=\sqrt{\sum_{l=1}^{M}\left(\mu_{l}-\lambda_{l}\right)^{2}}
$$

$d_{e}$ is a confirmed unknown parameter. If is used as the measurement for classification, theoretically it should eliminate the effect of randomness. However the values of $\vec{\mu}$ and $\vec{\lambda}$ can not be acquired directly, and we can just obtain the estimation of $d_{e}$ through estimating $\vec{\mu}_{\text {and }} \vec{\lambda}$.

$$
\text { Suppose: } \tilde{d}=D(\overrightarrow{\bar{X}}, \overrightarrow{\bar{Y}})=\sqrt{\sum_{l=1}^{M}\left(\bar{X}_{l}-\bar{Y}_{l}\right)^{2}}
$$

$\tilde{d}=D(\overrightarrow{\bar{X}}, \overrightarrow{\bar{Y}})$ is the estimation of $d_{e}$ and meanwhile it is stable.

We discuss the performance of $\tilde{d}$ as follows:

Suppose:

$$
D\left(\vec{X}^{i}, \vec{Y}^{j}\right)=\sqrt{\sum_{l=1}^{M}\left(X_{l}^{i}-Y_{l}^{j}\right)^{2}}=f\left(w_{1}, w_{2}, \cdots, w_{M}\right)
$$

where $w_{l}=X_{l}^{i}-Y_{l}^{j}$.

$$
\begin{aligned}
& \text { Suppose } \quad\left\{\begin{array}{l}
X_{l}^{i}=\mu_{l}+\varepsilon_{l}^{i} \\
Y_{l}^{j}=\lambda_{l}+\eta_{l}^{j}
\end{array}\right. \text {, then } \\
& \left\{\begin{array}{l}
E\left(\varepsilon_{l}^{i}\right)=E\left(X_{l}^{i}-\mu_{l}\right)=\mu_{l}-\mu_{l}=0 \\
E\left(\eta_{l}^{j}\right)=E\left(Y_{l}^{j}-\lambda_{l}\right)=\lambda_{l}-\lambda_{l}=0
\end{array}\right.
\end{aligned}
$$

Suppose $\quad\left\{\begin{array}{l}E\left(\varepsilon_{l}^{i}\right)^{2}=\sigma^{2} \\ E\left(\eta_{l}^{j}\right)^{2}=\sigma^{2}\end{array}\right.$, where $\sigma$ is the mean value of $X_{l}^{i}$ and $Y_{l}^{j}$.

The mathematical expectation value of $\tilde{d}$ is described as:

$$
E(\tilde{d}) \approx d_{e}+\frac{\sigma^{2}}{2}\left(\frac{1}{s}+\frac{1}{t}\right) \sum_{l=1}^{M} \frac{\partial^{2} f}{\partial w_{l}^{2}}
$$

Mean square error is:

$$
\begin{gathered}
E\left(\tilde{d}-d_{e}\right)^{2} \approx\left(\frac{1}{s}+\frac{1}{t}\right) \sigma^{2} \sum_{l=1}^{M}\left(\frac{\partial f}{\partial w_{l}}\right)^{2}+\left[\frac{\sigma^{2}}{2}\left(\frac{1}{s}+\frac{1}{t}\right) \sum_{l=1}^{M} \frac{\partial^{2} f}{\partial w_{l}^{2}}\right]^{2} \\
\text { So: } \quad \lim _{s, t \rightarrow \infty} E\left(\tilde{d}-d_{e}\right)^{2}=0
\end{gathered}
$$

With the increase of $\mathrm{s}$ and $\mathrm{t}, \tilde{d}$ will get closer to $d_{e}$, which shows that $\tilde{d}$ is an asymptotically unbiased estimation of $d_{e}$. So $\tilde{d}$ will serve as a very promising classifier.

\subsubsection{Weighted Euclidean distance classifier}

Considering that the randomness has different effects on each dimension of the feature vector, we use the weighted Euclidean distance to design the classifier:

$$
\hat{d}=\sqrt{\sum_{l=1}^{M}\left(\frac{\bar{X}_{l}-\bar{Y}_{l}}{\sigma_{l}}\right)^{2}}
$$

where $\sigma_{l}$ are the variance of $X_{l}^{i}$ and $Y_{l}^{j}$.

Also we can prove that $\hat{d}$ is an asymptotically unbiased estimation of $d_{e}$.

\section{Experiments and results}

To testify this method, we carry out the experiments as follows: we use handwriting samples from 100 persons. For each handwriting sample in the database, 20 texture images are constructed and feature extraction is implemented, after which we use equation (16) to get the estimation of $\vec{\lambda}$; For each handwriting for test, 20 texture images are constructed and equation (15) is used to estimate the mathematical expectation values $\vec{\mu}$. Experiments are implemented by using classifier described in equation (18) and equation (25) respectively. Then we enlarge the database to 500 persons and redo the experiments. All the results are shown in Table2.

Compared with Table 1, it can be easily found that through constructing a number of texture images to estimate the features, the identification accuracy improves a lot and exciting results are achieved both in the data set with handwriting samples collected from 100 persons and in the data 
set with handwriting samples collected from 500 persons.

Table 2. Experimental results of identification

\begin{tabular}{|c|c|c|c|}
\hline Results & Top 1 & Top 5 & Top 10 \\
$\begin{array}{c}\text { 100 persons } \\
\text { texture images } \\
\tilde{d}\end{array}$ & $64 \%$ & $90 \%$ & $98 \%$ \\
$\begin{array}{c}100 \text { persons } \\
20 \text { texture images } \\
\hat{d}\end{array}$ & $64 \%$ & $93 \%$ & $98 \%$ \\
\hline $\begin{array}{c}500 \text { persons } \\
20 \text { texture } \\
\text { images } \\
\tilde{d}\end{array}$ & $59 \%$ & $87 \%$ & $95 \%$ \\
\hline $\begin{array}{c}500 \text { persons } \\
20 \text { texture } \\
\text { images } \\
\hat{d}\end{array}$ & $59 \%$ & $89 \%$ & $95 \%$ \\
\hline
\end{tabular}

\section{Conclusions}

In this paper we present an approach for the construction of texture images which can be automatically operated by the computer. Then FFT is used to extract textural features. After the feature fusion, the stable spectrum features are derived and finally the Euclidean distance classifier is applied for handwriting identification. The experimental results show that this method has eliminated the effects of randomness so that it improves the identification accuracy remarkably.

This method is carried out both in the data sets with handwriting samples from 100 persons and handwriting samples from 500 persons. It achieves the highest accuracy of $98 \%$ and $95 \%$ concerning Top-10 respectively. Compared with the method with only constructing one single textural image, this method has improved the identification accuracy to a large extent. Our method has practical feasibility and holds the promise of concrete applicability in the data sets with large handwriting samples. The way that the texture images are constructed is rather convenient and simple. Although the computation cost is a little higher, the substantial progress in hardware performance can definitely deal well with it. In general the method based on stable spectrum features proposed in our paper is a rather promising writer identification method on the condition that the hand-written samples are with sufficient amounts of written Chinese characters.

The method proposed in our paper has been applied to the system of handwriting identification based on internet, which has been successfully applied in several cities.

\section{References}

[1] Said H E S, Tan T N, Baker K D, "Personal identification based on handwriting", In: Pattern Recognition, pp. 149-160, 2000, 33(1).

[2] Zhu Y, Wang Y, Tan T N, "Biometric personal identification based on handwriting", In: Proceedings of the $15^{\text {th }}$ International Conference on Pattern Recognition, Barcelona, pp.801-804, 2000.

[3] Ohanian P P, Dubes R C, "Performance evaluation for four classes of textural features [J]", In: Pattern Recognition, pp. 819-833, 1992, 25(6).

[4] Plamondon R, Lorrete G, "Automatic signature verification and writer identification -- the state of the art", In: Pattern Recognition, pp.107-131, 1989, 22(2).

[5] Zhu Yong, Tan Tieniu, Wang Yunhong, "Writer identification based on texture analysis", In: ACTA Automatica Sinica, pp. 229-234, 2001.17(2).

[6] Huang Ya-pin, Luo Si-wei, Chen En-yi, "Writer recognition based on independent component analysis", In: Journal of Chinese Information Processing, pp. 52-58, 2003.17(4). (In Chinese)

[7] Yang Zi-hua, Wu Min, Lin Jiang-rong, "Handwriting Identification System Based on Texture Analysis", In: Journal of Hunan Institute of Engineering, pp. 67-69, 2004.14(2). (In Chinese)

[8] Liu Hong, Li Jintao, Cui Guoqin, Tang Sheng, "Writer Identification using support vector machines and texture features", In: Journal of Computer-aided Design \& Computer Graphics, pp.1479-1484, 2003.15(12). (In Chinese)

[9] Liu Chenglin, Liu Yingjian, Dai Ruwei, "Writer identification by multi-channel decomposition and matching", In: ACTA Automatica Sinica, pp. 56-62 1997, 23(1).

[10]Liu Chenglin, Dai Ruwei, Liu Yingjian, "Modified Wigner distribution and application to writer identification", In: Journal of Computers, pp: 1018-1023, 1997, 20(11). (In Chinese)

[11]Yi Dong, Chen Qinghu, "Handwriting verification based on multiple classifiers", In: Computer Applications, pp.172-173, 2006, 26(1).(In Chinese) 